\title{
Un ingénieur et sa pratique. Les techniques et la subjectivité
}

An engineer and his practice. Technology and subjectivity

\section{Yves Cohen}

\section{(2) OpenEdition}

\section{Journals}

Édition électronique

URL : http://journals.openedition.org/dht/892

DOI : $10.4000 /$ dht.892

ISSN : 1775-4194

Éditeur :

Centre d'histoire des techniques et de l'environnement du Cnam (CDHTE-Cnam), Société des élèves du CDHTE-Cnam

\section{Édition imprimée}

Date de publication : 1 juin 2008

Pagination : 77-210

ISBN : 978-2-95-30779-1-9

ISSN : 0417-8726

\section{Référence électronique}

Yves Cohen, «Un ingénieur et sa pratique. Les techniques et la subjectivité », Documents pour l'histoire des techniques [En ligne], 15 | $7^{\text {er }}$ semestre 2008, mis en ligne le 18 octobre 2010, consulté le 08 septembre 2020. URL : http://journals.openedition.org/dht/892 ; DOI : https://doi.org/10.4000/dht. 892 


\title{
Un ingénieur et sa pratique. Les techniques et la subjectivité
}

\author{
Yves Cohen \\ École des hautes études \\ en sciences sociales
}

Cette publication est dédiée à F., grâce à qui tout ceci a commencé dans les années 1970.

e document présenté est écrit en pleine occupaLtion, à la fin de 1941, dans la Franche-Comté alors zone interdite. Son auteur est le directeur général des Fabrications et des Services techniques des Automobiles Peugeot. II occupe ce haut poste depuis 1917 avec une interruption de quelques années (de la fin 1922 au début de 1928) qu'il passe dans les usines Citroën à des fonctions de responsabilité, jusqu'à en devenir en 1927 le directeur adjoint des Fabrications. " J'ai attendu ce jour pour écrire mon histoire, qui est un peu celle de la fabrication de l'automobile, afin de montrer ce qui peut se passer dans les coulisses d'une industrie », écrit-il dans l'introduction'. Cette phrase dit tout : I'auteur, Ernest Mattern, né en 1880, diplômé des Arts et Métiers, école de Châlons, entre d'emblée, à son premier poste en 1900, dans l'industrie automobile pour n'en plus sortir. La première automobile ne date que de onze ans. Deux ans après avoir écrit ces mémoires, il doit quitter son poste sous la pression de l'occupant. Il est sur le champ nommé membre du conseil d'administration des Automobiles Peugeot (il échappe de peu à la déportation en 1944, tandis que plusieurs de ses directeurs sont déportés et que I'un d'eux y meurt). II décède peu d'années après la fin de la guerre, en 1952. Ce que la phrase citée dit, c'est à quel point Mattern identifie toute sa vie au développement de l'automobile. Plus même, que sa vie résume « un peu » cette histoire.

Merci à la famille d'Ernest Mattern pour sa généreuse autorisation de publier ce document.

1 Mattern E., Exemple vécu de la formation d'un ingénieur d'usine, Sochaux, dactyl., 1941, p. 4.
Ces mémoires et les archives qui l'environnent ont servi de source majeure pour une thèse de doctorat que j'ai soutenue en 1981 à l'université de Franche-Comté2. Mattern est devenu depuis un des personnages les plus connus de I'histoire de I'automobile en France. Son activité chez Citroën est relatée par Sylvie Schweitzer ${ }^{3}$. Pour I'historien italien de I'automobile Duccio Bigazzi, Mattern s'est révélé « probablement le plus pénétrant des organisateurs de la production dans l'automobile en France entre les deux guerres ${ }^{4}$. Or, selon ce que Mattern rapporte ici, c'était déjà ce que pensaient de grands hommes de l'automobile naissante en France comme Louis Delage, constructeur d'automobiles et coureur célèbre qui le classe en 1922 parmi les trois " meilleurs hommes de l'automobile » avant de le recommander en personne à son ami André Citroën ${ }^{5}$. Mattern a donc quelques titres à écrire la phrase citée. Pourtant, il faut aller plus loin et noter que ce qu'elle dit, c'est aussi l'affirmation d'un point de vue personnel sur cette histoire : le texte que nous offre Mattern ne cherche en rien à dissimuler sa subjectivité et plus encore sa certitude d'avoir eu, le plus souvent, raison contre tous. Le point de vue subjectif n'est aucunement masqué. La dernière phrase de l'introduction se lit ainsi : « Mon histoire est tellement extraordinaire qu'elle peut paraître invraisemblable. Il existe heureusement des témoins ou

2 Cohen Y., Ernest Mattern, les Automobiles Peugeot et le Pays de Montbéliard industriel avant et pendant la Guerre de 1914-1918. Composition sur une pratique d'organisateur, thèse de $3^{\text {e }}$ cycle, Université de Franche-Comté, Besançon, 1981, 557 p.

3 Schweitzer S., Des engrenages à la chaîne. Les usines Citroën, 1915-1935, Lyon, Presses Universitaires de Lyon, 1982, 204 p.

4 Bigazzi D., « Strutture della produzione : il Lingotto, I'America, I'Europa », dans I/ Lingotto, 1915-1939 dans C. Olmo dir., L'architettura, l'immagine, il lavoro, Turin, Umberto Allemandi \& C., 1994, p. 281.

5 Mattern E., op. cit., p. 66. 
des manuscrits qui peuvent certifier que tous les faits que je mentionne sont rigoureusement exacts. C'EST LA RÉFÉRENCE SOLIDE SUR LAQUELLE JE M'APPUIE POUR SOUTENIR LES OPINIONS ÉMISES EN TÊTE DE CE MÉMOIRE, SACHANT QUE N'IMPORTE LEQUEL DE MES CONTRADICTEURS NE POURRA RASSEMBLER UNE TELLE SÉRIE DE RÉUSSITES. DONC, J'AI RAISON. »

Mattern n'écrit pas pour être publié. Ce texte est rédigé à l'intention des dirigeants et des cadres supérieurs de Peugeot. II est suffisamment répandu dans I'entreprise pour qu'on en trouve des échos dans sa presse pendant et après la guerre. Apparemment, ces mémoires ont aussi été confiés à des journalistes spécialistes de l'automobile. L'intention de Mattern est d'appuyer des propositions de réforme et surtout de fonder son exigence de redresser des mesures jugées injustes prises à l'encontre de cadres proches de lui, sinon de lui-même. Dans une lettre à un cadre de rang équivalent au sien, Paul Vincent, directeur des Achats des Automobiles Peugeot, il écrit en 1942 : « J'ai écrit ma petite histoire, puisque je ne me suis jamais fait valoir pour montrer que ma réussite continue envers et malgré tous méritait de me laisser les coudées franches et de ne pas m'agacer dans mon travail. Je me suis décidé à l'écrire beaucoup pour les patrons et un peu pour vous ${ }^{7}$. " Même porté par une volonté farouche de justification, cet écrit est à usage interne et, de ce fait, soumis à une contrainte de vérité. Celle-ci s'exprime à de nombreuses reprises dans le texte: Mattern cite plusieurs fois les témoins des faits qu'il rapporte. Encore plus souvent, il indique qu'il possède les manuscrits des textes qu'il évoque. Le souci d'exactitude factuelle n'a d'égal que la subjectivité de l'écrit : il est très grand. Les choses n'en sont pas facilitées pour I'historien mais ces précautions garantissent au lecteur une histoire personnelle vraie, en quelque sorte.

À quelques exceptions près, malheureusement, les documents justificatifs évoqués par Mattern ont disparu. Le directeur avait rassemblé dans sa maison de Montbéliard une vaste documentation formée de fragments de son matériel de travail quotidien. Or c'est dans cette maison que la Gestapo a choisi de s'installer au cours de son repli après la libération de Paris en août. Ce n'est qu'en novembre que Montbéliard a été à son tour libéré et la Gestapo a alors incendié la « maison Mattern » pour ne pas s'embarrasser de ses propres archives,

6 lbid., p. 4 (la graphie d'origine a été respectée).

7 Archives Mattern [ensuite AM], Cahier de correspondance 1, Mattern à Vincent, 28 mars 42 (les soulignements et la ponctuation sont de Mattern). brûlant ainsi celles de leur hôte involontaire. Impossible dès lors de confronter l'autobiographie professionnelle de Mattern à ces documents qui ont servi pour l'écrire. D'autres documents rassemblés après la guerre et surtout la documentation historique mise désormais par les Automobiles Peugeot, grâce à leur association L'Aventure Peugeot, à la disposition des historiens, permettent de composer le nécessaire environnement documentaire d'un texte aussi fort. Les archives publiques, industrielles ou policières y aident aussi, de même que la presse, les documents syndicaux et I'histoire orale pour autant qu'elle demeure possible.

II n'en reste pas moins que ce texte n'a pas d'équivalent pour son époque. Mattern occupe un poste comparable à ceux de grandes personnalités qu'il connaît et qu'il a fréquentées. C'est le cas de Lucien Jannin (18771936, Arts et Métiers de Châlons 1892) qui accomplit chez Renault exactement ce que lui-même réalise chez Peugeot $^{8}$. Jannin est le premier chef de Mattern quand celui-ci débute après son diplôme chez Turgan et Foy, un constructeur de machines les plus diverses, à vapeur et à pétrole, pour la route, le rail ou l'eau, et où il rencontre aussi Delage. C'est encore le cas de Louis Guillot (Arts et Métiers de Lille 1901) qui est le directeur des fabrications de Citroën, dont il met au point toutes les installations de production comme la fabrication de 40000 obus shrapnell par jour pendant la guerre de 1914-1918 et celles qui permettront à Citroën de caracoler en tête des constructeurs durant tout l'entredeux-guerres ${ }^{9}$. Mattern travaille au plus près de Guillot durant tout son passage chez Citroën durant les années 1920. C'est aussi le cas de Ludovic Flandrin, rencontré également chez Turgan et Foy, qui fonde sa propre société d'automobiles avec Auguste Doriot, un transfuge de Peugeot. Nul document équivalent aux mémoires de Mattern pour tous ces acteurs majeurs de l'automobile, ni non plus d'ailleurs pour les entrepreneurs qui les emploient. Le texte qui pourrait le plus s'en rapprocher serait l'ouvrage écrit par Charles Sorensen, I'homme de la production de Ford, concepteur de la première chaîne de montage et longuement collaborateur très proche d'Henry Ford. Mais son ouvrage de souvenirs est d'un tout autre caractère que celui de Mattern. II est

\footnotetext{
8 Lepage E., « Lucien Jannin, mon patron », De Renault Frères à Renault Régie nationale, n²7, déc. 1983, pp. 96-97.

9 Picard F., « Les gadzarts dans l'industrie automobile », École Nationale Supérieure des Arts et Métiers, Paris, IPF, 1969, p. 149 ; Wolgensinger J., André Citroën, Paris, Flammarion, 1991, p. 240.
} 
écrit avec un spécialiste de l'écriture. Si certains récits sont très précis et forment un témoignage important à confronter comme tout autre à des sources d'origines différentes, comme celui de la conception de la chaîne de montage opérée depuis 1908 et seulement introduite pour la Ford T en 1913, on n'a pas le grand détail de l'action que fournit le texte de Mattern, la précision de ses confrontations avec des situations très diverses et de la mise en place de solutions plus ou moins complexes en tous domaines de l'activité industrielle ${ }^{10}$.

\section{L'organisation du document}

Avant d'aller plus avant dans ce que ce texte nous dit a) sur la technique, b) sur la pratique, c) sur les relations ouvrières du management, et enfin d) sur la reconnaissance de la pensée pratique, quelques mots sur sa lecture.

Une première difficulté vient de l'identification du titre. La couverture porte: Principes à observer pour la direction d'une société avec, en sous-titre, Exemple vécu de la formation d'un ingénieur d'usine. Seul ce dernier titre est repris sur la page de garde avec, cette fois, un autre sous-titre : Mes débuts dans l'industrie. Voici l'organisation effective du texte :

Les Principes à observer pour la direction d'une société ne sont en fait que l'introduction du texte, appelée « Objet de ce mémoire », de la p. 1 à la p. 4 bis.

Exemple vécu de la formation d'un ingénieur d'usine doit donc être considéré comme le titre général de cette autobiographie professionnelle. Ce que couvre cet énoncé est la majeure partie, divisée en trois sections principales mais très inégales en volume:

«Mes études», p. 5 ;

«Mes débuts dans l'industrie », p. 5 à 10 ;

«Mes travaux comme ingénieur praticien d'usine », p. 11 à 114. Ce titre passablement hérakléen englobe lui-même trois «parties »:

$1^{\text {e }}$ partie : Société Peugeot. Août 1906 - Octobre 1922, p. 11 à 65 ;

2e partie : Société Citroën. Novembre 1922 - Février 1928, p. 66 à 91 ;

3e partie : Société Peugeot. Février 1928, p. 92 à 114 (cette partie couvre en fait la séquence 19281941).

10 Sorensen Ch. E., avec Williamson S. T., My forty years with Ford, New York, Norton, 1956, 345 p. (rééd. Detroit, Wayne State University Press, 2006).
Une brève partie conclusive occupe les pages 114 et 115 .

Dans le recueil original, le texte principal de 115 pages est suivi de deux annexes qui ne sont pas reproduites ici : la première, annoncée dans le texte p. 64, est un mémoire de 16 pages sur la « Nouvelle organisation des services techniques » datant de juillet 1921 et la seconde, mentionnée p. 90, est un « Résumé des 246 questions pour la conduite des Usines de Forge et Fonderie (Citroën) » de 12 pages écrites au début de 1928.

\section{Plonger dans les techniques}

Cette autobiographie évoque ce que dit Stendhal du livre de l'architecte romain du xvie siècle Domenico Fontana dans Promenades dans Rome, à la date du 12 juillet 1828 : «Ainsi que les ouvrages des hommes qui ont agi, celui-ci est plein d'idées, et l'auteur ne songe pas au style ». Aussi bien est-elle composée de récits d'actes sur quarante années de pratique industrielle. L'importance de cette narration est due à la façon dont d'emblée, jeune ingénieur, Mattern envisage une carrière qui le plonge dans l'automobile dès son premier emploi. Identifiant sa volonté aux modes de réalisation du profit industriel, son approche des problèmes de l'entreprise s'opère délibérément par l'atelier, par la maitrise pratique de la technique du véhicule, des techniques de la production et des méthodes de commandement. Au succès dans l'abaissement des prix de revient, pierre d'angle, il subordonne un souci constant de l'augmentation des salaires du personnel placé sous ses ordres. En suivant pas à pas, acte par acte, les manifestations concrètes de l'idée qu'il se fait dès l'origine de sa profession, rigoureuse, intransigeante, poussée à ses dernières conséquences, se dégage la connaissance de l'unité des processus de production et de la pratique d'organisation au lieu de l'élaboration - au dire de Taylor lui-même - de la nouvelle organisation du travail : I'atelier. Puis le chemin nous fait accéder au niveau de I'usine à partir de 1912 et du groupe d'usines après 1917 et jusqu'en 1941, en passant par un cortège de redressements de situation menés dans l'immense empire industriel de Citroën, autrement nommé à l'époque le «Ford français».

L'évocation du nom de Taylor n'est pas un hasard. Nous sommes bien à l'époque taylorienne comme à l'époque fordienne de l'expérience industrielle. Plus largement encore, nous sommes bien dans la période où 
se forme la puissance du paradigme productif : I'investissement immense, à une échelle globale, du monde industriel dans la pensée organisationnelle et managériale fait de la sphère productive le lieu le plus intense de conception de normes pour l'action au $x x^{e}$ siècle ${ }^{11}$. Tandis que la pensée organisationnelle s'inspirait au XIX ${ }^{\mathrm{e}}$ siècle des pratiques administratives de l'État ${ }^{12}$, c'est I'inverse qui se produit au $x x^{e}$ siècle et encore en ce début du $x x^{e}{ }^{e}$ siècle : les administrations publiques s'inspirent des expériences des entreprises pour rationaliser leurs pratiques de gestion. Or l'automobile est le lieu central de la formulation de ces principes pour l'action organisée durant la majeure partie du $x x^{e}$ siècle ${ }^{13}$. Le texte de Mattern tient parole. II nous fait plonger, par le récit le plus souvent à la première personne, dans ce laboratoire des normes pratiques que sont l'atelier et I'entreprise automobiles. Mattern se montre en grand ordonnateur de la circulation de références théoriques, en passeur et adaptateur de principes et de traits d'expérience dans la conception de modes d'organisation locaux, situés. Mattern, s'il n'a presque pas voyagé en personne hors de France, connaît ses références et celles-ci perdent leur nom au profit de résolutions de problèmes infiniment répétées, spécifiques, toujours différentes et dont l'accumulation finit par former, en même temps qu'un paysage industriel, des séries de pratiques ancrées dans les choses et dans les corps.

Le texte nous immerge dans la technique, dans la logique d'un engagement passionné pour la technique. On rencontre à chaque détour le commerce avec des pièces, des dessins, des outillages, des calculs, des installations, des organisations, des dossiers, des fiches, des personnes. Ces personnes sont tout autant des ouvriers, des responsables d'entreprises extérieures, des collègues ingénieurs, des patrons. De grands noms sont évoqués au passage, ceux des Peugeot, certes, Robert et Jean-Pierre surtout, ceux d'André Citroën et de Louis Renault, ceux de personnages importants de l'automobile mondiale comme Alfred Sloan, le directeur général de la General Motors, qui a opposé avec succès une

11 Tolliday S. dir., The rise and fall of mass production, Cheltenham, Elgar, 1998, 2 vol., 842 p.; Zeitlin J. et Herrigel G. dir., Americanization and its limits : reworking US technology and management in postwar Europe and Japan, Oxford, Oxford University Press, 2000, $410 \mathrm{p}$

12 Cohen Y., «Fayol, un instituteur de l'ordre industriel », Entreprises et histoire, $\mathrm{n}^{\circ} 34$, décembre 2003, pp. 29-67.

13 Moutet A., Les Logiques de l'entreprise. La rationalisation dans l'industrie française de l'entre-deux-guerres, Paris, Éditions de l'EHESS, 1997, 495 p. imagination administrative à l'imagination productive d'Henry Ford et que Mattern croise à la fonderie Citroën de Clichy qu'il dirige en 1927. Passent aussi les noms de beaucoup d'ouvriers promus à des postes plus ou moins importants, d'ingénieurs spécialistes de l'étude des produits ou de la production, de « commis » devenus responsables d'importants services.

Ce que nous nommons trop facilement d'un mot au singulier, la technique, apparaît alors comme un ensemble très composite de différentes techniques. Pas un seul objet dans ce document, matériel, pièce, machine, ensemble, véhicule, charpente de bâtiment, voie de chemin de fer... qu'on puisse considérer isolé, hors pratique : tous sont pris dans des pratiques techniques spécifiques où se rencontrent des matières, des savoirs, des personnes liées par de la hiérarchie ou des relations économiques, des formes d'engagement diverses. Ce récit de pratiques techniques signale clairement, en toute généralité, que tout objet technique contemporain ne peut être envisagé que dans l'organisation au sein de laquelle il est commercialisé, fabriqué, conçu. Et cette « organisation » relève elle-même de techniques qui renvoient à différents degrés de matérialité, de hiérarchisation, d'ordonnancement, d'amoncellement formel, de formes de coordination, de saisie par le nombre comptable, etc. L'autobiographie de Mattern comporte quelques morceaux de bravoure où la pratique technique multiforme est concentrée et saisie dans la dynamique d'une pratique d'organisateur. C'est le cas par exemple en 1913 quand Mattern, directeur de I'usine d'Audincourt, cherche à rendre interchangeables les pièces du modèle 153 (pp. 32-34), ou quand il met en place la fabrication excessivement complexe des obus de 155 pendant la Première Guerre mondiale (p. 40) ou encore pour la conception de la 201 en 1928, le modèle sorti en 1929 par lequel Peugeot devient un constructeur de très grande série (pp. 104-105) ${ }^{14}$.

Pour l'ingénieur ou quelque intervenant que ce soit, aucun objet technique qui soit détachable des pratiques qui le prennent pour objet à un titre ou à un autre, c'est certainement là une des principales leçons

14 Fridenson P., "Ford as a model for French car makers, 19111939", dans Bonin H., Lung Y. et Tolliday S. dir., Ford, 19032003. The European history, Paris, Plage, 2003, vol. 1, pp. 125152 ; Cohen Y., "La technique, son contexte et le travail : que nous dit la conception de la 201 Peugeot ? », dans Belhoste J.-F., Benoît S., Chassagne S. et Mioche Ph. dir., Autour de l'industrie, histoire et patrimoine. Mélanges offerts à Denis Woronoff, Paris, Comité pour I'histoire économique et financière de la France, 2004, pp. 425-448. 
de ce texte. Les chercheurs en sciences sociales ont désormais largement adopté ce point de vue, depuis I'histoire défendue aux États-Unis par un Thomas Hughes jusqu'à la sociologie des sciences et des techniques ${ }^{15}$.

On pourrait classer ces techniques en fonction de la nature des entités qui en sont à l'impact. La réflexion historique et philosophique a fourni de ces classements, comme celui de Friedrich von Gottl-Ottlilienfeld qui distingue en 1923 quatre sortes de techniques : la technique du réel - qui recouvre à peu près le sens conféré couramment à « technique » lorsque celle-ci s'applique au monde matériel -, la technique individuelle - celle que Foucault nommerait technique de soi -, la technique sociale - qui a pour objet les relations entre individus - et la technique intellectuelle - qui recouvre les techniques de la pensée dans leur plus grande généralité ${ }^{16}$. Michel Foucault reprend fugitivement d'Habermas une classification comparable de trois types de techniques, certainement dérivée de cette même tradition intellectuelle allemande de réflexion sur les techniques: techniques de production, techniques de signification ou de communication, techniques de domination, à quoi il ajoute les techniques de soi ${ }^{17}$. Cette partition est proche encore de celle de Steven Shapin et Simon Schaffer - techniques matérielles, techniques littéraires et techniques sociales ${ }^{18}$. La lecture de l'autobiographie de Mattern nous fait en effet rencontrer : - des tech-

15 Voir par exemple I'œuvre de Thomas P. Hugues, depuis Networks of power. Electrification in Western society, 18801930, Baltimore, Johns Hopkins University Press, 1983, 474 p. jusqu'à Human-built world. How to think about technology and culture, Chicago, University of Chicago Press, 2004, 212 p. Ou encore, parmi un grand nombre de titres, le fondamental MacKenzie D. et Wajcman J. dir., The social shaping of technology, Milton Keynes-Philadelphie, Open University Press, $2^{e}$ éd. augm. 1999, 462 p. et le suggestif Callon M. et Law J., «L'irruption des non-humains dans les sciences humaines : quelques leçons tirées de la sociologie des sciences et des techniques », Les limites de la rationalité, vol. 2. Les figures du collectif, Reynaud B. dir., Paris, La Découverte, 1997, pp. 99-118.

16 Von Gottl-Ottlilienfeld F., Wirtschaft und Technik, Tübingen, 1923, cité par Ropohl G., "La signification des concepts de " technique » et " technologie » dans la langue allemande », Cahiers STS (De la technique à la technologie), n 2, 1984, pp. 30-41.

17 Foucault M., "Sexualité et solitude » (1981), Dits et écrits, Paris, Gallimard, 1994, vol. IV, p. 171.

18 Shapin S. et Schaffer S., Leviathan et la pompe à air. Hobbes et Boyle entre science et politique, Paris, La Découverte, 1993, 457 p. et Shapin S., "Une pompe de circonstance. La technologie littéraire de Boyle », Culture technique, n 14, juin 1985, pp. 71-87. niques de la connaissance (avec usage de graphiques, les mises en fiches ou l'établissement de prix de revient prévisionnels, les différents modes de calcul, y compris ceux qu'on opère sur place et qui diffèrent de ceux qu'on opère à une table de travail dans un bureau ${ }^{19}$, l'emploi de scientifiques divers au sein de laboratoires plus ou moins éloignés de la fabrication), - des techniques sociales (techniques des relations avec les autres, collègues, subordonnés, supérieurs, ou encore salaires, embauche, négociation), - des techniques matérielles (extrêmement variées pour un technicien professionnel : du comportement des matériaux et des pièces à l'organisation de productions complexes liées à la conception des produits), - des techniques de soi enfin (qui règlent l'économie des déplacements, des efforts, la logique de l'identité en cours de construction, les degrés de l'engagement dans les différentes formes de I'action : du contact direct à l'usage de moyens différés ou medias, etc.). D'autres déclinaisons sont possibles: ainsi on pourrait non seulement distinguer ce qui passe par le langage de ce qui passe par le nombre, mais encore, au sein du langage, distinguer les techniques orales des techniques de l'écrit ${ }^{20}$; on pourrait encore enquêter sur ces techniques qui cherchent à prendre les pratiques dans des formes, comme les formes organisationnelles, ou bien encore dans des normes formalisées ${ }^{21}$.

D'autres cadres d'interprétation sont d'ailleurs disponibles, comme la sociologie de la traduction qui trouverait dans ce texte de Mattern un nombre infini d'occurrences : les tolérances de fonctionnement et d'usinage sont traduites en dessins industriels eux-mêmes retraduits en équipement de production, un trait de crayon sur la table à dessin ou un geste ouvrier sont traduits en profit ou en perte industriels (de façon anticipée ou a posteriori), le régime même des déplacements et des rencontres d'un ingénieur est traduit en prospérité de la firme, sinon de toute une branche industrielle ${ }^{22}$. Le paradigme des " outils de gestion »,

19 Voir sur ce point l'irremplaçable Lave J., "La qualité de la quantité », Culture technique, n 14 (Latour B. dir., Les vues de l'esprit), juin 1985, pp. 47-57.

20 Sur ce point, Gardey D., Écrire, calculer, classer. Comment une révolution de papier a transformé les sociétés contemporaines (1800-1940), Paris, La Découverte, 2008, 319 p.

21 Thévenot L., "Un gouvernement par les normes », dans Conein B. et Thévenot L. dir., Cognition et information en société, Raisons pratiques, n 8, 1997, pp. 205-242.

22 Akrich M., Callon M., Latour B. dir., Sociologie de la traduction: textes fondateurs, Paris, Presses de l'École des mines, 
conçu par les chercheurs en management, ne manque pas non plus d'intérêt pour ce qu'il signale les effets directs et indirects, recherchés ou inattendus, des techniques managériales. Les outils de gestion mis en place sont eux-mêmes à la fois matériels (dans ces mémoires, les magasins et leurs fiches circulantes par exemple), comptables (suivi des pièces et des ensembles, contrôle des approvisionnements, cahier spécial d'étude des prix de revient...) ou juridictionnels (I'appréciation portée par le directeur sur les contremaîtres et donc leurs primes dépendent des dépenses faites en frais généraux par ces derniers). Ces outils accroissent les coordinations à la fois matérielles et humaines de secteur en secteur, d'ateliers en bureaux ${ }^{23}$.

Ce texte n'est donc pas une technologie au sens classique du terme ${ }^{24}$ : il ne tient aucun discours surplombant sur les techniques. II est pourtant un très intense discours de technicien sur ses techniques et il ouvre de la sorte la possibilité d'un discours plus général sur les pratiques techniques ${ }^{25}$.

\section{La leçon de « I'ingénieur praticien d'usine »}

La leçon que Mattern délivre personnellement et de façon insistante dans ce texte se concentre en un vocable surprenant dans la littérature du temps sur l'organisation : la « pratique d'atelier». Le texte l'évoque à plusieurs reprises. C'est le thème principal des « Principes à observer pour la direction d'une Société » qui forment l'introduction. II s'agit de la « pratique des machines et des hommes », de ce " contact des ouvriers

\section{6, $303 \mathrm{p}$.}

23 Berry M., Une technologie invisible ? L'impact des instruments de gestion sur l'évolution des systèmes humains, Paris, École polytechnique (Centre de recherche en gestion), juin 1983, 93 p. Le terme d'instrument administratif ou de direction était utilisé depuis le début du xxe siècle : en France chez Fayol (Peaucelle J.-L. dir., Henri Fayol. Inventeur des outils de gestion. Textes originaux et recherches actuelles, Paris, Economica, 2003, 316 p.) et chez nombre d'auteurs américains (comme Clark W., Le graphique Gantt, instrument de direction, Paris, E. Langlois, 1926, 159 p., traduction de The Gantt chart. A working tool of management, New York, 1922).

24 Voir Guillerme J. et Sebestik J., " Les commencements de la technologie », Documents pour I'histoire des techniques, n 14 (nouvelle série), 2e semestre 2007, pp. 49-121.

25 Ces points sont développés dans Cohen Y., Organiser à l'aube du taylorisme. La pratique d'Ernest Mattern chez Peugeot, 19061919, Besançon, Presses universitaires franc-comtoises, 2001, $490 \mathrm{p}$. et du personnel subalterne (qui a fait perdre au bon ingénieur d'usine) l'usage du langage correct ». Cette « pratique d'atelier » de l'organisateur, animée par le plaisir « de faire triompher (son) point de vue envers et contre tous », est rigoureusement l'inverse, l'opposé de la volonté ouvrière de conserver le contrôle et la maîtrise des processus de production ${ }^{26}$. II s'agit donc tout à la fois d'un rapport à soi, d'un rapport aux autres et d'un rapport aux matérialités.

Or il ne faut pas considérer cette leçon comme intemporelle. Elle est datée et localisée. Elle est celle d'un ingénieur qui se concentre délibérément sur la production et qui le fait à une époque dotée de ce point de vue de plusieurs caractéristiques. Tout d'abord, le fordisme est à la source du grand déploiement du paradigme de la production : la production de masse permet de développer une consommation de masse qui est le salut du capitalisme ${ }^{27}$. La Ford T est l'objet technique emblématique de ce mouvement : I'objet, conçu en fonction de sa production la plus économique, doit s'imposer au consommateur. Mais dans le même temps, d'autres doctrines industrielles se rapportant à la grande entreprise prennent leur essor et d'abord aux États-Unis. C'est le cas de l'entreprise à départements multiples (différentes marques ou différents produits) qu'incarnent fortement dans I'automobile la General Motors et dans la chimie Du Pont ${ }^{28}$ : le centre de la réflexion est ici l'administration de l'entreprise. Par ailleurs, parallèlement à l'essor de la grande entreprise, les entreprises petites et moyennes des districts industriels trouvaient le chemin d'une prospérité maintenue ${ }^{29}$. L'insistance de Mattern porte sur la nécessité de la présence sur les lieux de la production (ce peut être tout autant le cubilot que la table à dessin) dans une logique productive - quand les pratiques de la firme décentralisée et départementalisée s'intéressent plutôt aux pratiques de

26 Voir Montgomery D., «Quels standards ? Les ouvriers et la réorganisation du travail aux États-Unis (1900-1920)», Le Mouvement social, $n^{\circ} 102$, janv.-mars 1978, pp. 101-127 et id., Workers' control in America: studies in the history of work, technology, and labor struggles, Cambridge, Cambridge University Press, 1979, $190 \mathrm{p}$.

27 On peut lire directement les propos du constructeur américain, entre autres dans Ford $\mathrm{H}$., Ma vie et mon œuvre, Paris, Payot, 1925, 314 p. et Ford H. et Crowther S., Aujourd'hui et demain, Paris, Payot, 1926, $348 \mathrm{p}$

28 Voir en particulier Chandler A. D. Jr, Stratégies et structures de l'entreprise, Paris, Les Éditions d'organisation, 1989, 543 p.

29 Sabel Ch. et Zeitlin J. dir., World of possibilities : flexibility and mass production in Western industrialization, Cambridge, Paris, Cambridge University Press, Éditions de la MSH, 1997, 510 p. 
la gestion à distance ${ }^{30}$. L'autobiographie de Mattern témoigne d'une incessante bataille sur ce point avec ses employeurs. À son grand dam, sa direction technique est déplacée en 1921 de la région de Montbéliard, où sont les usines, vers le siège de Peugeot à LevalloisPerret dans la proche banlieue parisienne, prélude à sa mise à l'écart. Nommé de nouveau directeur de la production en février 1928, il ramène sa direction à Sochaux dès septembre de la même année, y déménageant aussi le bureau d'études. La bataille se poursuit jusque dans les années 1940 contre des initiatives, soit de grands commis centraux comme le secrétaire général de la société Peugeot, soit de Jean-Pierre Peugeot en personne. C'est d'ailleurs en grande partie du fait de cette configuration qu'il faut comprendre la décision prise par Mattern d'écrire sa grande justification dans le texte ici publié. L'autobiographie vient montrer la nécessité impérieuse et toujours actuelle du contact du manager avec les matières et les hommes.

Et effectivement, Mattern passe le plus clair de son temps dans les ateliers et les usines. II programme ses parcours pour les visiter tous au cours de la semaine. ॥ en fait une leçon exemplaire pour ses directeurs, leur démontrant que le rendement dépend de leur présence. Comme le disent ses mémoires, ces parcours lui servent tout autant à voir et à comprendre qu'à résoudre des problèmes. C'est donc un procédé cognitif et un principe de la connaissance technique et organisationnelle en même temps qu'un procédé hiérarchique et une modalité de la décision ${ }^{31}$. Sorensen rapporte les descentes de Ford: " S'il avait un problème et qu'un ingénieur comme Farkas, Zoerlein ou Smith s'en occupait, il venait s'arrêter auprès d'eux pour jeter un coup d'œil et bavarder (for a look-see and a chat). " $^{32}$. Nous sommes ici au cœur de la pratique de gouvernement technique dans la grande organisation industrielle. II s'agit là d'une pratique constante du palabre au contact des problèmes qui se situent dans le rapport dynamique entre matières (pièces, machines, installations, usines)

30 Comme le théoricien français Henri Fayol dans son Administration industrielle et générale, Paris, Dunod, 1918, 156 p. (1ère éd. dans Bulletin de la Société de l'industrie minérale, $n^{\circ} 3$, 1916). Sur l'organisation de la distance, voir Yates J. A. , "Graphs as a managerial tool : a case study of Du Pont's use of graphs in the early twentieth century", Journal of business communication, vol. 22, n 1, 1985, pp. 5-33.

31 Cohen Y., «L'espace de I'organisateur: Ernest Mattern, 1906-1939 », Le Mouvement social, n 125, 1983, pp. 79-96.

32 Sorensen C., op. cit., p. 19. et hommes (ouvriers, maîtrise, techniciens, ingénieurs, directeurs) $)^{33}$

Or, sur la longue durée de l'histoire industrielle, c'est précisément le travail d'ingénieurs comme Mattern, avec au centre le palabre sur place, qui confère aux espaces productifs plus de régularité, de stabilité, de prévisibilité et qui permet précisément, progressivement, aux managers de quitter l'atelier, au contrôle budgétaire d'asseoir mieux sa prise sur la production, aux bureaux des méthodes de s'éloigner des lieux du travail effectif ${ }^{34}$. Les gestes entrent dans des machines de plus en plus automatiques et jusqu'aux robots, les calculs et en général les inscriptions se font plus précis, les normes du travail elles-mêmes se standardisent et s'universalisent $^{35}$. La prise sur le travail - ouvrier d'abord et de plus en plus technicien et managérial -, dans ses dimensions physiques et mentales comme dans le rapport à soi, se fait plus savante, plus investigatrice et plus envahissante.

La «pratique d'atelier » de Mattern suppose un engagement corporel et un régime de gestion de soimême dans le rapport à l'espace d'action extrêmement intenses. L'autobiographie en témoigne à chaque page. Elle montre ainsi comment se forment des unités plus ou moins stabilisées qui lient l'organisateur-directeur et son espace d'action en tant qu'il est formé d'installations, de machines, de personnes, de problèmes ou, pour employer un autre vocabulaire, d'humains, de non humains et $d^{\prime}$ « affaires $»^{36}$. Le récit de la réforme de I'usine de Beaulieu de 1917 à 1920 est à cet égard remarquable ${ }^{37}$. La conception de la 201, modèle, usines, main-d'œuvre, logements dans le même mouvement, n'est pas moins démonstrative : ils sont le corps de

33 Voir le très suggestif Orr J. E., Talking about machines : an ethnography of a modern job, Ithaca, ILR Press, 1996, 172 p.

34 Guigueno V., L'Éclipse de l'atelier. Les missions françaises de productivité aux États-Unis dans les années 1950, mémoire de DEA (sous la dir. d'Antoine Picon), ENPC, 1994, 108 p. ; Berland N., Le contrôle budgétaire, Paris, La Découverte, 2002, 120 p. et id., Mesurer et piloter la performance, Bruxelles, PricewaterhouseCoopers, Paris-La Défense, Éd. de la Performance, 2004, 230 p. ; Hatzfeld N., Les gens d'usine. 50 ans d'histoire à Peugeot-Sochaux, Paris, Les Éditions de l'atelier, 2002, 598 p.

35 Chatzis K., "Searching for Standards: French engineers and time and motion studies of industrial operations in the 1950s", History and technology, vol. 15, n 3, 1999, pp. 233-261.

36 Sur le « corps étendu » de l'ingénieur, voir le passionnant Mialet H., L'entreprise créatrice. Le rôle des récits, des objets et de l'acteur dans l'invention, Paris, Lavoisier, 2008, 201 p.

37 Mattern E., Exemple vécu, op. cit., pp. 59-64. 
Mattern comme chaque unité produite emporte une parcelle du corps des opérateurs de toute sorte.

Ceci dit sur l'engagement impressionnant de la personne dans son action, les deux pages de conclusion indiquent de leur côté que cette « mise de soi » implique une éthique réfléchie. Le partage est très exactement effectué entre la sphère privée et la sphère professionnelle. Les formes intenses de la présence personnelle de Mattern dans I'usine excluent toute pratique relevant de la cité « domestique » au sens que lui donnent Luc Boltanski et Laurent Thévenot ${ }^{38}$ : on ne peut être plus que Mattern dans l'effort d'instaurer un régime « industriel », au sens de ces mêmes sociologues, c'est-àdire un monde orienté exclusivement sur l'efficacité et le profit industriel, sans arrangement subreptice, aussi circonscrit soit-il, avec l'ordre domestique. C'est aussi dans cette dimension qu'il faut comprendre sa revendication de ne s'être jamais « mis en avant » dans le discours public sur le développement de Peugeot et en particulier son rôle dans la sortie de la 201. Ceci s'opère paradoxalement par un engagement personnel extraordinaire qui marque de son sceau les équipements mis en place, les habitudes de travail attachées au lieu et aussi les produits. C'est l'usine Sochaux de Mattern, celle qui est reconçue en 1928, qui a duré jusque dans les années 1980, compacifiée sans doute, densifiée, étendue jusqu'aux limites permises par la vie urbaine. Pesanteur des lieux, des matérialités, des actes humains pris dans ces matérialités : seule, à partir des années 1980, la nouvelle époque de production a pu quitter ce Sochaux par l'abandon de l'ancienne usine et la construction de nouveaux bâtiments conformes à d'autres principes de fabrication, précisément parce que la décision a été prise de s'arracher à soixante ans d'habitudes industrielles, de mode de formation des produits comme des relations humaines et des profits ${ }^{39}$. Et en fin de compte, cette autobiographie qu'il écrit comme outil dans une bataille renouvelée pour ses conceptions de la gestion industrielle, il la laisse aussi aux historiens en une sorte d'opération qui permet à terme d'effacer son propre effacement : I'auteur de ces lignes ne cache pas qu'il s'est prêté à cette dimension des mémoires.

38 Boltanski L. et Thévenot L., De la justification. Les économies de la grandeur, Paris, Gallimard, 1991, 485 p.

39 Beaud S. et Pialoux M., Retour sur la condition ouvrière. Enquête aux usines Peugeot de Sochaux-Montbéliard, Paris, Fayard, 1999, 468 p. ; Durand J.-P. et Hatzfeld N., La chaîne et le réseau. Peugeot-Sochaux, ambiances d'intérieur, Lausanne, Éd. Page Deux, 2002, 303 p. ; Hatzfeld N., op. cit.

\section{Reconnaître la peine ouvrière et concevoir la chaîne}

II y aurait tant à dire encore sur de très nombreux aspects importants de ce texte. Beaucoup de pages pourraient être écrites en particulier sur ce qu'il évoque des relations ouvrières de cet ingénieur de production. Jamais Mattern n'est pris en défaut d'attention au sort de la main-d'œuvre ouvrière. Voici un texte écrit peu après 1936 pour la maîtrise de Sochaux. Le Pays de Montbéliard a connu de brèves grèves qui ont rompu I'atmosphère de consentement à la philanthropie patronale qui vient pourtant d'être récemment confirmée par l'élection d'un Peugeot au siège de député tenu jusque-là par un socialiste, à rebours du mouvement qui fait passer le Front populaire en France :

"Dès l'instant que la production marche, vous devez et nous devons nous ingénier et nous torturer l'esprit sans cesse et sans arrêt pour que la vie à l'usine soit rendue à tous la plus agréable possible. Nous devons avoir pour notre personnel toutes les gentillesses possibles. II ne faut pas qu'en rentrant à l'usine les ouvriers se disent : "Nous rentrons au bagne", mais qu'ils se disent : "Puisque le travail est une nécessité sociale, c'est encore chez Peugeot qu'on est le mieux." II faudrait être idiot pour prétendre fabriquer de bonnes voitures avec des ouvriers mécontents. Une belle machine avec un ouvrier dans un mauvais état d'esprit fournira des pièces moins précises qu'une mauvaise machine conduite par un ouvrier content de sa situation. (...) Voilà maintenant le problème que nous voudrions résoudre : supprimer les mécontents à l'usine et faire en sorte que, à part les 4 à $5 \%$ d'intransigeants qui nous critiquent toujours de parti pris, tous soient contents d'être chez Peugeot ${ }^{40}$. "

Dans son livre de 1925, Mattern s'était fait le théoricien d'une politique souple face aux grèves ouvrières : dans une grève pour les salaires, ne pas maintenir de position intransigeante ; au contraire, ne faire aucune concession s'il s'agit d'une grève qui se rapporte à I'organisation du travail ou aux méthodes de direction (point évoqué ci-dessus par la formule : « Dès l'instant que la production marche... ») ; s'il s'agit d'une grève contre des sanctions, étudier si celles-ci sont justes ou non et les annuler dans le second $\operatorname{cas}^{41}$. Mattern a ef-

40 AM, Ernest Mattern, Pourquoi j'ai créé le questionnaire des chefs d'équipe, 26 février 1937. Sur la maîtrise, voir Kopff A. La maîtrise au Centre de production Peugeot-Sochaux (1945-1975), mémoire de DEA, Paris, EHESS, 1993, $111 \mathrm{p}$.

41 Mattern E., Création, organisation et direction des usines, 
fectivement une réputation de justice. Le propos du journal communiste local au retour de Mattern en 1928 est sans équivoque à un moment où le parti communiste est très isolé du fait de son sectarisme : « Ernest Mattern revient comme directeur technique au plaisir des ouvriers. Les huiles font un de ces nez...42 ${ }^{42}$ Lorsque quelques mois plus tard, le même journal dénonce le plan de concentration des usines sur Sochaux avec son accompagnement de logements ouvriers pour familles, pour célibataires et pour étrangers, il parle de « village nègre », de " camp de concentration » et $d^{\prime}$ " " esclavage moderne » sans toutefois attaquer nommément Mattern ${ }^{43}$. Des propos élogieux seront encore tenus sur lui par la presse de la résistance proche des communistes.

Mattern est bien I'un des plus talentueux concepteurs de la chaîne de montage. Celle-ci ne se met pas en place sans des discours comme celui qui vient d'être cité. Ainsi pour Ford, la répétition des mouvements ne cause aucun préjudice physique. Pour Robert Peugeot, le travail à la chaîne dégage l'esprit des ouvriers justement grâce à la répétitivité des gestes ${ }^{44}$. De tels discours sont des composantes de la technique. Techniques euxmêmes (relevant de la rhétorique, technique de persuasion et la plus noble des techniques pour les Grecs), ils I'accompagnent indéfectiblement. Les tayloriens ne manquent jamais de dire qu'ils maîtrisent la question de la fatigue ouvrière ${ }^{45}$. Ils calculent les charges, adoptent des limites, insèrent des temps de repos dans leur reconstitution concentrée et épurée des séquences de travail. Mais fondamentalement, ils oublient le principe d'Armand Imbert, ce médecin qui s'est spécialisé à la charnière du $x x^{e}$ siècle dans la physiologie du travail, qui veut que ce soit le travailleur qui possède le meilleur point de vue possible sur son travail ${ }^{46}$.

Paris, Dunod, 1925, 306 p. (2e éd. 1926).

42 Le Semeur, 11 février 1928.

43 Le Semeur, 19 octobre 1929.

44 Ford H., Ma vie et mon œuvre, op. cit., p. 120. Peugeot R., L'organisation du travail et des loisirs, Paris, "La Cause », 1934, $16 \mathrm{p}$.

45 Lahy J.-M., Le système Taylor et la physiologie du travail professionnel, Paris, Masson et Cie, 1916, 196 p. ; Vatin F., Organisation du travail et économie des entreprises, Paris, Éditions d'organisation, 1990, 203 p. ; Rabinbach A., Le moteur humain, l'énergie, la fatigue et les origines de la modernité, Paris, La Fabrique, 2004, $555 \mathrm{p}$.

46 Le Bianic Th. et Vatin F., "Armand Imbert (1850-1922), la science du travail et la paix sociale », Travail et emploi, n 111, juillet-septembre 2007, pp. 7-19.
Ce faisant, Mattern fabrique la société industrielle, celle du travail en miettes, du travail décomposé, des ouvriers non qualifiés, des gestes répétitifs, de la fatigue physique et morale. Certes, ce n'est pas là son intention. N'hésitons pas devant le truisme : I'enfer est pavé de bonnes intentions. En travaillant pour le profit capitaliste (I'autobiographie dit qu'il en a reçu la leçon sur sa planche à dessin dès ses premiers pas dans l'industrie. II en fait plus tard un principe d'éducation pour la maîtrise et les techniciens), il pense aider à constituer une société juste où le travail est rétribué, où le progrès permet d'alléger la durée et la charge du travail. II ne s'agit pas seulement là d'une question de rapport entre I'intention et ce qui est fait effectivement. Construire I'usine du travail "simplifié », déqualifié pour obtenir les meilleurs rendements, grâce à une intensification technique due à l'emploi de plus en plus d'ingénieurs, était accompagné, devait être accompagné d'un discours de progrès pour tous. Quelle que soit I'attention à la « peine » ouvrière, manifeste chez cet ingénieur, I'organisateur ne peut penser à la place de ceux qui en font l'épreuve.

\section{Un ingénieur qui ne pense pas ou une pensée de praticien?}

Quelle histoire faire avec ces documents ? Pour saisir la logique de cette pratique, on ne peut pas se servir d'une sociologie qui partirait de pratiques déjà sociales et s'imposant aux personnes comme des normes ou des règles. Une histoire analytique et réflexive qui se repaît de singularités convient mieux, mais pour chercher de façon plus efficace un sens qui ne se trouve que dans la mise en série d'expériences voisines, dans la recherche de régularités non postulées à l'avance. Dès lors, les documents exceptionnels comme ceux de Mattern permettent d'interroger d'autres documents qui le sont moins et de reconstruire des collectifs et des collectivités. Par exemple, Mattern, même s'il a écrit un livre sur l'organisation et le management ${ }^{47}$, n'a jamais pris place parmi les auteurs donnés pour théoriciens de l'organisation.

Tout grand organisateur qu'il fût, il reste un homme de l'automobile. C'est avec les hommes de l'automobile qu'il convient de le comparer, de mettre son activité en série à la fois pour identifier ce qu'est cette activité d'organisateur qui a joué un si grand rôle au

47 Mattern E., Création..., op. cit. 


\section{Un ingénieur et sa pratique. Les techniques et la subjectivité}

$x x^{e}$ siècle et pour saisir la place spécifique de cet organisateur-ci au sein de cet ensemble. Sur le premier de ces deux points : I'automobile s'est construite comme le domaine industriel-roi du $x x^{e}$ siècle comme l'a été parallèlement la physique pour la science. Les raisons en sont en premier lieu le caractère mixte de cette industrie : tout à la fois industrie de main-d'œuvre et industrie intense en capital, elle a dès les premières années du $x x^{e}$ siècle été prise dans une dynamique d'intensification technique accélérée, comme le royaume de la mécanisation et de l'automatisation pour la production en masse d'objets mécaniques complexes. En second lieu, mais inséparablement du premier, l'objet automobile s'est imposé au marché comme un besoin fondamental pour la construction d'une vie quotidienne correspondant à une société se dessinant comme de consommation - révolutionnant tout à la fois le marché et le paysage des pays conquis. Objet fétiche de l'individualisme moderne où un produit industriel propriété de l'individu transporte celui-ci en tous lieux avec une infinie flexibilité : la théorie de Ford visant à rendre consommateur le producteur n'a pas joué un petit rôle en ce sens. La révolution automobile est une révolution de la production comme une révolution des usages et du monde des « choses banales ${ }^{48}$. Un troisième point, lui-même inséparable des deux autres, est que l'automobile a été le lieu emblématique du travail déqualifié : la chaîne de montage qui, depuis Ford, est devenue la condition de l'alliance entre production de masse et consommation de masse est le système d'un travail répétitif et dépourvu de sens pour ses opérateurs, le plus souvent choisis parmi des jeunes hommes recrutés au loin, parmi des femmes et des immigrés. Les OS (ouvriers spécialisés), héros des luttes ouvrières des années 1960 et 1970, dont les revendications sont mal prises en charge par des syndicats qui s'appuient plus volontiers sur les ouvriers professionnels, sont les produits de la chaîne de montage comme l'est la voiture standard des années 1910-198049.

Dans ce paysage, le groupe de Sochaux a pleinement sa place ainsi que son concepteur industriel, Ernest Mattern. Le Sochaux conçu par Mattern, accompagné des savoirs et des « routines » correspondants,

48 Bardou J.-P., Chanaron J.-J., Fridenson P. et Laux J. M., La révolution automobile, Paris, Albin Michel, 1977, 385 p. ; Roche D., Histoire des choses banales. Naissance de la consommation, XVII -XIX siècles, Paris, Fayard, 1997, $350 \mathrm{p}$.

49 Vigna X., L'insubordination ouvrière dans les années 68. Essai d'histoire politique des usines, Rennes, Presses universitaires de Rennes, 2007, 378 p. assure à la firme une durable solidité. Le directeur peut se prévaloir d'avoir formé un personnel éduqué aux " hauts rendements » et bâti Sochaux, " I'usine qui est organisée pour gagner de l'argent si on travaille en série sur des programmes simples $»^{50}$. Avec le Sochaux du plan de 1928 et de la 201, on a un ensemble industriel apte à produire en grande série avec des coûts maîtrisés ménageant de larges marges aux actionnaires - ce qui est très exactement l'opposé de l'ensemble de fabrication un moment si efficace et puissant construit par André Citroën sur le quai de Javel et dans une couronne d'usines autour de lui. Sochaux ne forme rien d'autre que les fondations de la prospérité de la marque pour une soixantaine d'années, celles du fordisme, qui sont celles où le paradigme productif domine ${ }^{51}$. L'usine, vue comme complexe de pratiques de travail, contribue à bâtir la supériorité de Peugeot sur Citroën qui conduit à terme au rachat du second par le premier en $1973^{52}$. Si I'on se conforme au vocabulaire standard de la business history (ou histoire des entreprises), Mattern se révèle un parfait middle manager en proposant à Peugeot cette grand unité de production à « efficacité d'échelle minimale », c'est-à-dire à la taille opérationnelle nécessaire pour atteindre le coût unitaire le plus bas $»^{53}$.

Deux éléments : les capacités installées, dans leur matérialité mécanique et architecturale - qui sont pliées à l'organisation -, et les compétences humaines spécifiques - pliées aux normes et aux lieux : on a là une version particulière des " capacités organisationnelles » (organizational capabilities) de Chandler. Celles-ci forment un intéressant concept précisément parce qu'elles regroupent l'espace et la connaissance, autrement dit le matériel et le cognitif, pour en faire une valeur et un trait qui expliquent la puissance économique ${ }^{54}$. Les

50 AM, Cahier de correspondance, Mattern à Jordan, 16 février 1942.

51 Voir le dernier ouvrage en date sur Sochaux : Belot R. et Lamard P., Peugeot à Sochaux. Des hommes, une usine, un territoire, Panazol, Lavauzelle, 2007, 372 p.

52 Loubet J.-L., Automobiles Peugeot, une réussite industrielle, 1945-1974, Paris, Économica, 1990, 469 p. et id., Histoire de I'automobile française, Paris, Seuil, 2001, 569 p.

53 Chandler A. D. Jr, Organisation et performance des entreprises, tome 1. Les USA, 1880-1948, Paris, Les Éditions d'organisation, 1992, p. 52 sq.

54 Chandler A. E., " L'évolution historique de la grande entreprise industrielle: structures et capacités organisationnelles », Entreprise et histoire, $n^{\circ} 10,1995$, p. 15 ; voir aussi Organisation et performances..., op. cit. Ce concept est utile à condition d'en respecter la formation: il comporte à la fois les compétences spécifiques de l'organisation et, c'est le point, les installations 
deux éléments ne sont pas étrangers l'un à l'autre : la conformation du capital installé à l'organisation relève certes d'un travail savant, mais les installations contribuent à leur tour à la formation des compétences. L'espace organisé se fait organisateur. II a une action.

Avec cet ingénieur, si fortement attaché à I'automobile que son action contribue à conformer le contexte de son développement, tout autant que le développement de l'automobile est le contexte de son action, se profile l'opposé de personnalités à peu près équivalentes qui ont joué un rôle marqué et remarqué dans les débuts de l'automobile industrielle en France. On pense à Georges de Ram qui a tenté la première expérience de taylorisation, en 1907-1908, en cherchant à introduire certaines méthodes de Taylor dans un atelier de machines-outils de Renault ${ }^{55}$. II reste chez Renault jusqu'en janvier 1913 chef d'atelier de machines-outils où il dirige 400 ouvriers et son action est sans doute à l'origine de la série de grèves contre le chronométrage qui saisit cette usine en 1912 et 1913, conférant à la nouvelle discipline américaine de management un parfum de scandale ${ }^{56}$. Or de Ram ne paraît pas particulièrement intéressé par l'automobile. Dès septembre 1908, il prend directement contact avec Taylor. Ce qui frappe, aussi bien dans l'article qu'il publie en 1909 que dans sa correspondance avec Taylor, c'est que ses expériences auraient pu tout aussi bien se dérouler dans

conformées par l'organisation et spécifiées, soit le capital installé. La notion de capacités organisationnelles est trop souvent réduite, dans la littérature managériale et historienne, à la seule dimension de la connaissance ou de la compétence, dans l'empressement d'oublier ou de négliger la dimension matérielle et technique. L'inflexion présentée est double : elle insiste sur l'effet d'espace sur les compétences (et non pas seulement sur le mouvement inverse) et elle comprend dans les « compétences »- toujours valorisées - les modalités du travail gouvernées et contrôlées par des normes - souvent oubliées et négligées aussi-, pas seulement pour les ouvriers spécialisés mais pour I'ensemble du personnel.

55 De Ram G., «Quelques notes sur un essai d'application du système Taylor dans un grand atelier de mécanique français ", Revue de métallurgie, sept. 1909, pp. 929-933. La première traduction d'un texte de Taylor paraît en 1907 dans la Revue de métallurgie. Elle est celle de son œuvre fondamentale, La direction des ateliers (1903).

56 Fridenson P., Histoire des usines Renault, tome 1, Naissance de la grande entreprise, 1898-1939, Paris, Seuil, 1998 (1éd. 1972), p. 73 et Frederick W. Taylor Collection (Stevens Institute of Technology, Hoboken, New Jersey), doss. 180, de Ram à Taylor, 2 mars 1913) ; Pouzet P.-L., Les Gadzarts pionniers de I'automobile jusqu'en 1914, Lyon, Fondation de l'Automobile Marius Berliet, 1983, p. 261. une autre industrie que l'automobile. Aucune allusion, par exemple, à la question décisive de l'interchangeabilité qui préoccupe en priorité un Mattern appuyé sur son expérience du montage à l'usine Peugeot de Lille. L'interchangeabilité entraîne la mise en place de travaux spécifiques dans les ateliers de machines-outils et d'outillage, comme la fabrication de montages d'usinage et celle d'instruments de vérification, et aussi l'organisation particulière du contrôle. De Ram ne s'en fait nullement l'écho. D'un autre côté, il est prêt à quitter Renault qui ne fournit pas, selon lui, de bonnes conditions à une application correcte du système Taylor, et à réorganiser les ateliers navals des Chantiers Normands, au Havre, pour chercher ensuite d'autres entreprises où introduire le système ${ }^{57}$. Ainsi, c'est I'organisation scientifique qui passionne de Ram, et peu importe sur la production de quoi il l'exerce, tandis que Mattern affiche une conception de l'organisation étroitement spécifiée dans l'automobile. II continue ensuite, semble-t-il, une carrière d'ingénieur-conseil tout à fait en dehors du monde automobile ${ }^{58}$. Il en est de même pour Charles de Fréminville, longtemps directeur technique des usines Panhard-Levassor, pionnières de I'automobile au même titre que Peugeot. L'ingénieur est progressivement mis à l'écart. II reconstitue son activité comme expert en management scientifique jusqu'à participer à l'organisation du Congrès international de I'Organisation scientifique du travail qui se tient à Paris en 192959 .

57 Taylor Collection, doss. 180, lettres de De Ram à Taylor, en particulier 12 octobre 1909 et 12 octobre 1910. G. de Ram, op. cit.

58 Agnès d'Angio signale notre homme au Creusot et chez d'autres mécaniciens comme la Société française de constructions mécaniques et la Société alsacienne de constructions mécaniques («La mutation des structures de la société Schneider et Cie entre 1898 et 1914. Parallélisme avec les débuts de la diffusion des idées tayloriennes en France», dans Croyances et gestion, Toulouse, Presses de l'Université des sciences sociales de Toulouse, 2000, p 260).

59 Voir laux J. M., In first gear. The French automobile industry to 1914, Liverpool, Liverpool University Press, Montréal, McGillQueen's University Press, 1976, XIII-239 p. et parmi de nombreuses publications: de Fréminville Ch., « Les méthodes scientifiques de travail dans I'industrie. Le système Taylor », La Technique Moderne, tome vIII, suppl. au n 6, 15 mars 1914, xvı p. ; aussi Danzin A. dir., Charles de Fréminville, 1856-1936, pionnier de l'organisation scientifique du travail, sl, Aubin Éditeur, 2000, 190 p. ; Cayet Th., Organiser le travail, organiser le monde : étude d'un milieu international d'organisateurs-rationalisateurs durant l'entre-deux-guerres, thèse $d$ 'histoire, Florence, European University Institute, 2005. 
De la sorte, si l'organisation du travail devait être un « champ » intellectuel où la notoriété se mesure à la production de théories dites «pensée », alors Mattern est tout à fait invisible, contrairement aux deux auteurs précédents et à bien d'autres. Un de mes excellents collègues historiens, très féru d'organisation, à qui j'avais fait lire le document ici publié, m'a d'ailleurs dit dans les années 1980 à propos de Mattern : «Ce qu'il y a d'extraordinaire, c'est qu'il ne pense pas. » Spontanément, même la tradition historienne informée consistait alors à ne pas mettre de la pensée dans les gestes et les pratiques, surtout s'ils étaient « techniques ». La tendance s'est inversée depuis. Le même historien a sûrement oublié son propos d'alors et ne le répéterait pas. De la pensée est reconnue même dans le geste qu'on disait naguère mécanique ou automatique ${ }^{60}$. À plus forte raison chez un ingénieur, comme Mattern, qui n'a cessé de réfléchir à son activité, dans son cours même (rapports, notes, lettres) ou a posteriori dans des écrits autobiographiques orientés sur son action comme les présents mémoires ${ }^{61}$. Permettez-moi de signaler, à l'inverse, une des surprises de mes rencontres d'après thèse : ce commentaire de Michel Foucault me disant, après la lecture de mon travail doctoral sur la pratique de Mattern, que j'avais fait de I'histoire de la pensée. Grâce aux promoteurs généreux des Documents pour I'histoire des techniques, j'espère par cette publication rendre plus visibles ces penseurs praticiens de la technique et leurs élaborations, en suivant la tradition illustrée par exemple par l'ouvrage d'Hélène Vérin sur les ingénieurs $d^{\prime}$ autres siècles ${ }^{62}$.

\section{Conclusion}

Ce document dit quelque chose à l'histoire des techniques. Cet apport est dû à la fois à ceci qu'il est contraint par la vérité (témoins vivants, documents disponibles) et totalement subjectif : il parle d'actes techniques et en même temps du rapport qu'entretient l'acteur avec ses actes $^{63}$. Ce n'est pas seulement que la technique

60 Voir les travaux des ergonomes et par exemple Theureau J., Le cours d'action. Méthode développée, Toulouse, Octarès, 2006, $384 \mathrm{p}$.

61 ou Document autobiographique (sans titre), Sochaux, 28 février 1947, 22 p. dactyl.

62 Vérin H., La gloire des ingénieurs. L'intelligence technique du XVI $\left.\right|^{\mathrm{e}}$ au XvII siècle, Paris, Albin Michel, 1993, 455 p.

63 Cohen Y., « Ernest Mattern et la 201 Peugeot : intensifications techniques et subjectivité », dans Belot R., Cotte M. et Lamard P. dir., La technologie au risque de l'histoire, Sévenans et se montre indissociable du social : histoire et sociologie ont déjà fait justice de la séparation et personne n'affirme plus qu'on puisse étudier les techniques sans chercher des relations sociales qui contribuent à les constituer ${ }^{64}$. L'expérience d'un ingénieur aussi imprégné de sa propre activité technique, innovant sans cesse tant en matière de produit, de production que de gestion, intervenant dans la stratégie de la firme dont il n'est qu'un grand commis, montre des techniques, aussi matérielles qu'elles soient, indétachables des personnes et de leurs pratiques partagées, communes, conflictuelles souvent, empreintes en tout cas de subjectivité ${ }^{5}$. Cette subjectivité pratique ne signifie aucunement un repli de l'étude ou de la réflexion sur les individus et sur l'individualité. Au contraire, sa reconnaissance rend possible plusieurs choses : I'étude de ce que sont les formes de l'engagement personnel dans la technique et plus encore de ce que cet engagement en personne fait aux techniques (aux objets, aux usines, au travail, aux techniques de gestion, aux techniques sociales, etc.) ; la réflexion sur des pratiques partagées, dans leurs proximités et leurs écarts ; l'étude analytique de régularités: identité ou différence des préoccupations pratiques comme le temps, l'espace, le produit, la production, le flux, les savoirs, la place ménagée pour les humains et leurs initiatives, la place du nombre, celle des matérialités, etc. Ou la série : il est différent de produire des automobiles pour un marché de classes moyennes et de produire des paquebots ${ }^{66}$. Ou la sécurité : c'est un souci relativement mineur pour les fabricants d'automobiles de ce temps, tout à la fois pour le produit et la production, tandis qu'il en est un grand pour les gens des mines. Et ces préoccupations s'entremêlent, s'appuient les unes sur les autres, toutes portées de façon différenciée par des acteurs aux positions variées dans l'espace pratique. Partir d'une ana-

Paris, Université de Technologie de Belfort-Montbéliard et Berg International Éditeurs, 2000, pp. 147-163.

64 Staudenmaier J., Technology's storytellers: reweaving the human fabric, Cambridge, MIT Press, 1985, 350 p.; Akrich M., «Comment sortir de la dichotomie technique/société. Présentation des diverses sociologies de la technique ", dans Latour B. et Lemonnier P. dir., De la préhistoire aux missiles balistiques, Paris, La Découverte, 1994, pp. 105-131.

65 Voir sur les Seguin, Cotte M., Le Choix de la révolution industrielle. Les entreprises de Marc Seguin et de ses frères (18151835), Rennes, Presses universitaires de Rennes, 2007, 572 p. 66 Dewerpe A., « L'exercice des conventions. Le cas de l'industrie navale française (fin xixe-début xxe siècle) », dans Lepetit B. dir., Les formes de l'expérience, Paris, Albin Michel, 1995, pp. 99126. 
lyse de telles pratiques, en s'appuyant sur les gestes, les normes qui les gouvernent et les mots qui les nomment, permet enfin de reconsidérer la pensée de la domination. L'autobiographie professionnelle de Mattern fournit une remarquable initiation à cette considération des pratiques techniques. Elle invite à relancer l'analyse sur d'autres expériences, sur d'autres objets, à sortir de la prééminence de l'automobile dans les sciences sociales de la technique et du travail au $x x^{e}$ siècle. 